\title{
Styrylpyridinium Derivatives as New Potent Antifungal Drugs and Fluorescence Probes
}

\section{OPEN ACCESS}

Edited by:

Miguel Cacho Teixeira, University of Lisbon, Portugal

Reviewed by:

Rajendra Prasad,

Jawaharlal Nehru University, India Slawomir Milewski,

Gdańsk University of Technology, Poland

*Correspondence: Marie Kodedová marie.kodedova@fgu.cas.cz

Specialty section: This article was submitted to Antimicrobials, Resistance and Chemotherapy,

a section of the journal

Frontiers in Microbiology

Received: 17 April 2020 Accepted: 06 August 2020

Published: 28 August 2020

Citation:

Vaitkienè S, Daugelavičius $R$, Sychrová H and Kodedová M (2020) Styrylpyridinium Derivatives as New

Potent Antifungal Drugs and Fluorescence Probes.

Front. Microbiol. 11:2077.

doi: 10.3389/fmicb.2020.02077

\author{
Simona Vaitkiené1, Rimantas Daugelavičius ${ }^{1}$, Hana Sychrová ${ }^{2}$ and Marie Kodedová*
}

${ }^{1}$ Department of Biochemistry, Faculty of Natural Sciences, Vytautas Magnus University, Kaunas, Lithuania, ${ }^{2}$ Laboratory of Membrane Transport, Division Biotechnology and Biomedicine Centre of the Academy of Sciences and Charles University (BIOCEV), Institute of Physiology of the Czech Academy of Sciences, Vestec, Czechia

The incidence of Candida glabrata infections increases every year due to its higher resistance to commonly used antifungal drugs. We characterized the antifungal mechanism of action of eight new styrylpyridinium derivatives, with various $\mathrm{N}$-alkyl chains $\left(-\mathrm{C}_{6} \mathrm{H}_{13},-\mathrm{C}_{8} \mathrm{H}_{17},-\mathrm{C}_{10} \mathrm{H}_{21},-\mathrm{C}_{12} \mathrm{H}_{25}\right)$ and different substituents, on C. glabrata strains differing in their drug resistance due to the presence or absence of two major drug-efflux pumps. We found that the tested styrylpyridinium compounds affected the growth of $C$. glabrata cells in a compound- and strain-dependent manner, and apparently they were substrates of $\mathrm{CgCdr1}$ and $\mathrm{CgCdr2}$ pumps. Further, we determined the impact of the tested compounds on plasma membrane integrity. The ability to cause damage to a plasma membrane depended on the compound, its concentration and the presence of efflux pumps, and corresponded well with the results of growth and survival tests. We also tested possible synergism with three types of known antifungal drugs. Though we did not observe any synergism with azole drugs, styrylpyridinium compounds $\mathbf{5}$ and $\mathbf{6}$ together with FK506 demonstrated excellent antifungal properties, whereas compounds $\mathbf{2}, \mathbf{3}, \mathbf{5}$, and $\mathbf{6}$ exhibited a significant synergistic effect in combination with terbinafine. Based on our results, derivatives $\mathbf{2}$ and $\mathbf{6}$ turned out to be the most promising antifungal drugs. Moreover, compound $\mathbf{6}$ was not only able to effectively permeabilize the yeast plasma membrane, but also exhibited significant synergism with FK506 and terbinafine. Finally, we also characterized the spectroscopic properties of the tested styrylpyridinium compounds. We measured their absorption and fluorescence spectra, determined their localization in yeast cells and found that their fluorescence characteristics differ from the properties of current commercial vacuolar styrylpyridinium markers and allow multi-color staining. Compounds $\mathbf{1}, \mathbf{3}, \mathbf{7}$, and $\mathbf{8}$ were able to accumulate in plasma and vacuolar membranes, and compounds $\mathbf{2}, \mathbf{5}$, and $\mathbf{6}$ stained the whole interior of dead cells. In summary, of the eight tested compounds, compound $\mathbf{6}$ is the most promising antifungal drug, compound $\mathbf{8}$, due to its minimal toxicity, is the best candidate for a new vacuolar-membrane probe or new benchmark substrate of $C$. glabrata $C d r$ pumps, and derivative $\mathbf{5}$ for a new vital dye.

Keywords: styrylpyridinium derivatives, Candida glabrata, diS- $\mathrm{C}_{3}(3)$ assay, membrane potential, yeast, multidrug resistance, vacuolar marker 


\section{INTRODUCTION}

Infections caused by fungal pathogens have become a critical health problem, especially for immunocompromised individuals. Even though Candida albicans persist as the most common species causing invasive candidiasis, the number of non-albicans (particularly C. glabrata) infections is intensively increasing worldwide every year. The rising incidence of C. glabrata infections occurs as a result of the higher resistance of $C$. glabrata to widely used antifungal drugs, such as azoles, polyenes, echinocandins, and flucytosine (Ksiezopolska and Gabaldón, 2018). The majority of current antifungal drugs target ergosterol (polyenes) and its biosynthetic pathway (azoles, allylamines, and morpholines). Azoles, but also allylamines, such as terbinafine, disturb the function of the yeast plasma membrane by inhibiting various enzymes in the ergosterol biosynthetic pathway, resulting in the depletion of ergosterol together with the accumulation of toxic sterols in the membrane (Petrikkos and Skiada, 2007). Nevertheless, the fungistatic action of azoles often evokes the development of resistance, for example C. glabrata exhibits higher innate and acquired resistance to the azole class of antifungals than C. albicans (Pais et al., 2019). This resistance is often associated with the overexpression of two multidrug resistance (MDR) transporters - $C g$ Cdr1 and $C g \mathrm{Cdr} 2$ (Cannon et al., 2009).

Increase in multidrug resistance is a serious problem, and a novel treatment for fungal infections is urgently needed. Finding new antifungal molecules could deal with the rising prevalence of C. glabrata. Promising drug candidates can be also searched for among compounds that are currently used for very different applications, e.g., the painkiller/antiinflammatory drug diclofenac potentiates the activity of caspofungin, while it attenuates the effectiveness of fluconazole against C. albicans (Mathé and Van Dijck, 2013; Urai et al., 2014). Likewise, lipophilic styrylpyridinium dyes (FM4-64 and FM1-43) were used for a long time as efficient fluorescence probes for staining and analyzing cell membrane features and pathologies (Heese-Peck et al., 2002; Bolte et al., 2004; Miner et al., 2019). Only a few studies demonstrated that some styrylpyridinium compounds may also have antibacterial effects (Wyrzykiewicz et al., 1994; Chanawanno et al., 2010). Therefore we focused on determining and comprehensively analyzing the antifungal mechanism of action of styrylpyridinium derivatives to evaluate their possible application in the treatment of fungal infections. In our previous study (Vaitkiené et al., 2020), we newly synthetized six styrylpyridinium compounds and, together with seven already known styrylpyridinium derivatives, investigated their fungicidal effect, synergism with fluconazole, and cytotoxicity to mammalian cells. Our results indicated that eight compounds were able to reduce $C$. albicans growth and some of them were substrates of $C$. albicans MDR pumps, since different concentrations were required to inhibit the growth of C. albicans strains with deleted $C D R 1, C D R 2$, or MDR1 genes (Vaitkiené et al., 2020).

The aim of this study was to characterize the mechanism of action of eight styrylpyridinium compounds, which were selected based on their highest efficiency against C. albicans in our previous study (Vaitkiene et al., 2020), on C. glabrata strains differing in their ability to export drugs. We used the potentiometric fluorescence probe $\operatorname{diS}_{-} \mathrm{C}_{3}(3)$ to evaluate the impact of these drugs on plasma membrane integrity and identify $\mathrm{CgCdr} 1$ substrates, since the $\mathrm{diS}_{-} \mathrm{C}_{3}(3)$ probe not only accumulates in yeast cells in response to membrane potential (Gášková et al., 1998), but is also a substrate of CgCdr1 (Kodedová and Sychrová, 2016), thus helping to identify other substrates of this drug-efflux pump via competitive inhibition among its substrates. We also monitored C. glabrata growth in the presence of styrylpyridinium derivatives and tested possible synergism with known antifungal drugs and substrates of $C g C d r 1$ and/or $C g C d r 2$ pumps. Further, we performed a basic spectroscopic analysis of these compounds and found possible applications of the styrylpyridinium derivatives with low toxicity as new fluorescence probes in yeast research.

\section{MATERIALS AND METHODS}

\section{Styrylpyridinium Derivatives}

Styrylpyridinium derivatives were synthesized according to a well-known two-step procedure involving the alkylation of $\gamma$-picoline with an appropriate alkyl bromide and further condensation with substituted benzaldehyde (Vaitkiene et al., 2020). Figure 1 illustrates the basic structure of styrylpyridinium derivatives, the particular substituents of these 8 compounds used in this study are summarized in Table 1. The compounds were dissolved in ethanol, usually as $2 \mathrm{mg} / \mathrm{mL}$ stock solutions.

\section{Yeast Strains and Growth Conditions}

Candida glabrata strains used in this study are listed in Table 2. Cells were grown at $30^{\circ} \mathrm{C}$ in YPD medium ( $1 \%$ yeast extract, $2 \%$ peptone, $2 \%$ glucose, and $2 \%$ agar for solid media). For experiments, cells from the early exponential growth phase were harvested, washed twice with sterile deionized water and resuspended in deionized water (for fluorescence microscopy) or in $10 \mathrm{mM}$ citrate-phosphate (CP) buffer ( $\mathrm{pH} 6.0$ ) to $\mathrm{OD}_{600}=0.2$.

\section{Determination of Plasma-Membrane Integrity [diS- $\mathrm{C}_{3}(3)$ Assay]}

The relative membrane potential and integrity of the yeast cell plasma membrane was estimated by a fluorescence assay based on the potential-dependent redistribution of the fluorescence probe diS- $C_{3}$ (3) (3,3'-dipropylthiacarbocyanine iodide) (Denksteinová et al., 1997; Gášková et al., 1998), as described in Kodedová et al. (2019). The probe was added to a final concentration of $0.02 \mu \mathrm{M}$ to yeast suspensions in CP buffer, and samples were occasionally

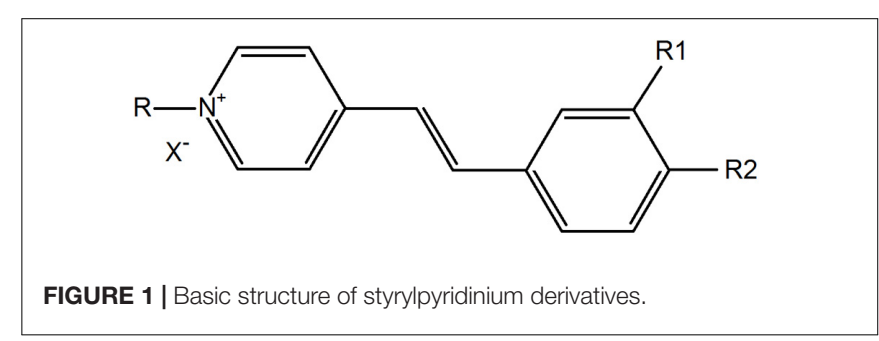


TABLE 1 | Styrylpyridinium derivatives used in this study.

\begin{tabular}{|c|c|c|c|c|c|}
\hline Compound & R1 & $\mathbf{R} 2$ & $\mathbf{R}$ & $\mathbf{X}^{-}$ & References \\
\hline 1 & $-H$ & $\mathrm{CH}_{3}-\mathrm{CH}-\mathrm{N}-\mathrm{CH}-\mathrm{CH}_{3}$ & $-\mathrm{C}_{12} \mathrm{H}_{25}$ & $\mathrm{Br}^{-}$ & $\begin{array}{l}\text { Vaitkienè et al., } \\
2020\end{array}$ \\
\hline 2 & $-H$ & $-\mathrm{OH}$ & $-\mathrm{C}_{12} \mathrm{H}_{25}$ & $\mathrm{Br}^{-}$ & $\begin{array}{l}\text { Wyrzykiewicz } \\
\text { et al., } 1994\end{array}$ \\
\hline 3 & $-\mathrm{O}-\mathrm{CH}_{3}$ & $-\mathrm{O}-\mathrm{CH}_{3}$ & $-\mathrm{C}_{12} \mathrm{H}_{25}$ & $\mathrm{Br}^{-}$ & $\begin{array}{l}\text { Vaitkienè et al., } \\
2020\end{array}$ \\
\hline 4 & $-\mathrm{H}$ & $-\mathrm{CN}$ & $-\mathrm{C}_{12} \mathrm{H}_{25}$ & $\mathrm{Br}^{-}$ & $\begin{array}{l}\text { Vaitkienè et al., } \\
2020\end{array}$ \\
\hline 5 & $-\mathrm{H}$ & $-\mathrm{OH}$ & $-\mathrm{C}_{8} \mathrm{H}_{17}$ & $\mathrm{Br}^{-}$ & Krieg et al., 2007 \\
\hline 6 & $-H$ & $-\mathrm{OH}$ & $-\mathrm{C}_{10} \mathrm{H}_{21}$ & $\mathrm{Br}^{-}$ & $\begin{array}{c}\text { Mishra et al., } \\
2001\end{array}$ \\
\hline 7 & $-H$ & $\mathrm{CH}_{3}-\mathrm{N}-\mathrm{CH}_{3}$ & $-\mathrm{C}_{8} \mathrm{H}_{17}$ & $\mathrm{Br}^{-}$ & $\begin{array}{l}\text { Shiraishi et al., } \\
2010\end{array}$ \\
\hline 8 & $-\mathrm{H}$ & $\mathrm{CH}_{3}-\mathrm{N}-\mathrm{CH}_{3}$ & $-\mathrm{C}_{6} \mathrm{H}_{13}$ & $\mathrm{CH}_{3}-\mathrm{C}_{6} \mathrm{H}_{4} \mathrm{SO}_{3}^{-}$ & $\begin{array}{c}\text { Dubur et al., } \\
1984\end{array}$ \\
\hline
\end{tabular}

TABLE 2 | Yeast strains used in this study.

\begin{tabular}{|c|c|c|}
\hline Strain & Genotype or phenotype & References \\
\hline DSY565 & C. glabrata clinical isolate, resistant to azoles & Sanglard et al., 1999 \\
\hline DSY1041 & $c d r 1 \Delta::$ hisG-URA3-hisG & Sanglard et al., 1999 \\
\hline DSY1612 & cdr2 $\Delta:: h i s G-U R A 3-h i s G$ & Sanglard et al., 2001 \\
\hline DSY1613 & cdr2 $\Delta::$ hisG cdr1 $1 \Delta:$ hisG-URA3-hisG & Sanglard et al., 2001 \\
\hline
\end{tabular}

gently stirred. Fluorescence emission spectra $\left(\lambda_{\text {ex }}=531 \mathrm{~nm}\right.$, $\lambda_{\mathrm{em}}=560-590 \mathrm{~nm}$ ) of the cell suspensions were measured in a FluoroMax-4 spectrofluorimeter (Horiba Scientific). The staining curves represented the dependence of the fluorescence emission maximum wavelength $\lambda_{\max }$ on the time of staining. The tested compounds were usually added after $\sim 10 \mathrm{~min}$ of staining. Representative results of at least three independent experiments (always with similar results) are shown.

\section{Estimation of Cell Survival (Plating Test)}

To determine cell viability, C. glabrata cells resuspended in CP buffer were treated with the styrylpyridinium derivatives with occasional gentle stirring for $20 \mathrm{~min} .10 \mu \mathrm{L}$ of cell suspensions were then diluted 100-fold, and aliquots were spread on YPD agar plates in triplicates. The number of colonies (CFU) was determined after 1 day of incubation of the plates at $30^{\circ} \mathrm{C}$. Experiments were repeated three times. Results are shown in the form of percentages as means $\pm \mathrm{SD}$; the control sample without exposure to the drugs was regarded as $100 \%$ cell survival.

\section{Growth Curves}

To compare the resistance of strains to styrylpyridinium derivatives, the growth in liquid medium was monitored in a 96-well plate reader Elx808 (BioTek) with shaking at $30^{\circ} \mathrm{C}$ for $24 \mathrm{~h}$. Cells were cultivated in $100 \mu \mathrm{L}$ of YPD medium inoculated to $\mathrm{OD}_{600}=0.001$. The $\mathrm{OD}_{600}$ was measured at $1 \mathrm{~h}$ intervals. Growth curves were obtained in duplicates over a broad range of drug concentrations $(0.5-20 \mu \mathrm{M})$. The plotted values of relative growth after $24 \mathrm{~h}$ cultivation are the means $\pm \mathrm{SD}$ of three independent experiments.

\section{Disk Diffusion Tests}

Disk diffusion tests were performed for estimating the synergistic effect of styrylpyridinium derivatives with FK506, terbinafine, fluconazole and ketoconazole. Washed yeast cells were diluted into YPD top agar ( $1 \%$ agar) to $\mathrm{OD}_{600}=0.2$ and poured onto solidified YPD plates (2\% agar). Drug solutions $(2 \mu \mathrm{L})$ at the concentrations indicated in the text were spotted onto paper disks laid on top of the solidified agar with cells. The plates were photographed after 1 day of incubation at $30^{\circ} \mathrm{C}$. Representative results of three independent experiments are shown.

\section{Measurement of Absorption and Fluorescence Spectra of Styrylpyridinium Derivatives}

The absorption spectra of $20 \mu \mathrm{M}$ styrylpyridinium derivatives in deionized water were measured in a quartz cuvette with an optical path length of $10 \mathrm{~mm}$ using an Agilent 8453 UV-visible spectrophotometer in the range 190-1100 nm. All spectra were corrected for the baseline. Maximum absorption wavelengths $\left(\lambda_{\mathrm{abs}}\right)$ summarized in Table 3 were used for the fluorescence excitation of the given compounds. The fluorescence emission spectra of $20 \mu \mathrm{M}$ styrylpyridinium derivatives in deionized water were measured in a quartz cuvette with a FluoroMax-4 spectrofluorimeter in the range 350-1000 nm. Fluorescence spectra were normalized to the unified maximum fluorescence intensity. The Stokes shift (Table 3) was calculated as the difference between the spectral position of the maximum of the absorption $\left(\lambda_{\mathrm{abs}}\right)$ and the maximum of the fluorescence emission $\left(\lambda_{\mathrm{em}}\right)$ expressed in wavelength units.

\section{Fluorescence Microscopy}

Washed C. glabrata DSY565 cells were resuspended in deionized water, stained with $2 \mu \mathrm{M}$ styrylpyridinium derivatives and after cca 10 min observed with a Leica TCS SP8 WLL 
TABLE 3 | Maximum absorption and fluorescence emission wavelengths and corresponding Stokes shifts of styrylpyridinium derivatives.

\begin{tabular}{lccc}
\hline Compound & $\lambda_{\text {abs }}(\mathbf{n m})$ & $\lambda_{\text {em }}(\mathbf{n m})$ & Stokes shift $(\mathbf{n m})$ \\
\hline $\mathbf{1}$ & 474 & 609 & 135 \\
$\mathbf{2}$ & 377 & 505 & 128 \\
$\mathbf{3}$ & 384 & 531 & 147 \\
$\mathbf{4}$ & 335 & 405 & 70 \\
$\mathbf{5}$ & 375 & 505 & 130 \\
$\mathbf{6}$ & 375 & 504 & 129 \\
$\mathbf{7}$ & 450 & 603 & 153 \\
$\mathbf{8}$ & 452 & 605 & 153 \\
\hline
\end{tabular}

SMD confocal fluorescence microscope (objective HC PL APO CS2 $63 \times / \mathrm{NA} 1.20$ with water immersion, $\lambda_{\mathrm{ex}}=405 \mathrm{~nm}$, green fluorescence $\lambda_{\mathrm{em}}=480-650 \mathrm{~nm}$, red fluorescence $\left.\lambda_{\mathrm{em}}=650-800 \mathrm{~nm}\right)$. For the demonstration of the different accumulation of styrylpyridinium derivatives in yeast cells with damaged and intact plasma membranes, C. glabrata cells were resuspended in deionized water to $\mathrm{OD}_{600}=0.2$ and treated with $3 \mu \mathrm{M}$ octenidine dihydrochloride (ODDC), causing full permeabilization of yeast cells within $15 \mathrm{~min}$ (Kodedová et al., 2019). Then the cells were washed with deionized water and used as a model of killed cells for styrylpyridinium staining.

\section{Styrylpyridinium Efflux Assay}

The styrylpyridinium compound $\mathbf{8}$ was added to a final concentration of $2 \mu \mathrm{M}$ to yeast suspensions $\left(\mathrm{OD}_{600}=0.2\right)$ in $\mathrm{CP}$ buffer, and samples were occasionally gently stirred. Fluorescence emission spectra $\left(\lambda_{\mathrm{ex}}=452 \mathrm{~nm}, \lambda_{\mathrm{em}}=500-750 \mathrm{~nm}\right)$ of the cell suspensions were measured in a FluoroMax- 4 spectrofluorimeter (Horiba Scientific). The excitation and emission slits were set to $4 \mathrm{~nm}$. The staining curves represented the time-dependence of the fluorescence intensity registered at emission maximum wavelength. The results are shown as means $\pm \mathrm{SD}$ of three independent experiments.

\section{Statistical Analysis}

The statistical analyses were performed with SigmaPlot 13 . ANOVA with subsequent post-hoc test was used for identifying significant differences.

\section{RESULTS AND DISCUSSION}

\section{Impact of Styrylpyridinium Derivatives on Growth of C. glabrata Strains Differing in the Number of Their Drug-Efflux Pumps}

First, we assessed the effect of compound addition on the growth of yeast cultures. We used a wild-type azole-resistant clinical isolate DSY565 and its three mutants lacking one or two MDR

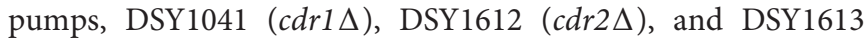
$(c d r 1 \Delta c d r 2 \Delta)$. The high antifungal resistance of DSY565 strain is caused by significant upregulation of CDR1, while CDR2 is only moderately expressed (at the basal level) in this strain
(Sanglard et al., 2001). Comparison of the growth inhibition among the four strains may suggest whether the used compounds are substrates of the two MDR pumps or not. We monitored the growth in YPD cultures in the presence of three different concentrations $(0.5,1$, and $2 \mu \mathrm{M})$ of compounds $\mathbf{1 - 8}$ (Figure $\mathbf{1}$ and Table 1) for $24 \mathrm{~h}$. The obtained results are summarized in Figure 2. All the tested compounds exhibited at least some inhibitory effect at the used range of concentrations except for compound 8, for which concentrations an order of magnitude higher $(5,10$, and $20 \mu \mathrm{M})$ were required to obtain any significant inhibition of C. glabrata growth (Figure $2 \mathbf{H}$ ). The growth of wild-type cells was the most inhibited by compounds 2 and 6, already at $1 \mu \mathrm{M}$ concentration (Figures 2B,F), a little bit less by compounds $\mathbf{1}, \mathbf{4}$, and $\mathbf{5}$ (significant inhibition visible at $2 \mu \mathrm{M}$ concentration, Figures $2 \mathrm{~A}, \mathbf{D}, \mathbf{E})$, and the presence of compounds 3 and 7 caused only a very slight decrease in growth when these two compounds were present at a concentration of $2 \mu \mathrm{M}$ (Figures 2C,G).

The inhibitory effect of almost all the tested compounds was more pronounced in the cultures of mutant cells. The only exception was compound 5, whose effects were quite similar for all 4 strains (Figure 2E). Cells lacking Cdr1 were more sensitive to all the tested compounds than the wild type, suggesting that they are probably effectively pumped by Cdr1 in the wild-type cells. The absence of Cdr1 was the most pronounced with compounds 2 and $\mathbf{6}$ (Figures 2B,F). The obtained results suggested that Cdr2 may also export the majority of the tested compounds, as the absence of both pumps in the double mutant led to the highest sensitivity of cells to all the tested compounds except compound 8 (Figure 2).

In summary, compounds $\mathbf{2}$ and $\mathbf{6}$ were the most toxic for wild-type cells and their toxicity was tremendously increased in the absence of Cdr pumps. Compound $\mathbf{8}$ was not significantly toxic to C. glabrata cells and compound 5, though toxic at the $2 \mu \mathrm{M}$ concentration, is probably not a good substrate of Cdr1 and Cdr2. When we compared the growth curves (not shown), we realized that styrylpyridinium derivatives affected their shape in a similar way, they predominantly prolonged the lag phase of growth curves (in the range of several hours) rather than slowing down the rate of exponential growth. Doubling times of wild-type and mutant strains cultivated in the presence of $0.5 \mu \mathrm{M}$ styrylpyridinium compounds differed on average of $5 \mathrm{~min}$, except more toxic compounds 2 and $\mathbf{6}$, which prolonged doubling times of some strains to 11 and $22 \mathrm{~min}$ (doubling times of $c d r 1 \Delta c d r 2 \Delta$ strain were not determined in the presence of compounds $\mathbf{1}, \mathbf{2}, \mathbf{6}$, and 7 , as cell cultures did not reach exponential growth phase during 24-h cultivation). The prolongation of lag phase could be explained by a transitional period necessary for cell adaptation to the presence of drugs (induction of expression of Cdr pumps) or by the immediate killing of a certain amount of inoculated cells upon their primary contact with styrylpyridinium compounds. Of course, both of the above may occur together. To elucidate whether the used compounds were killing cells rapidly, we investigated the changes in plasma-membrane integrity upon 

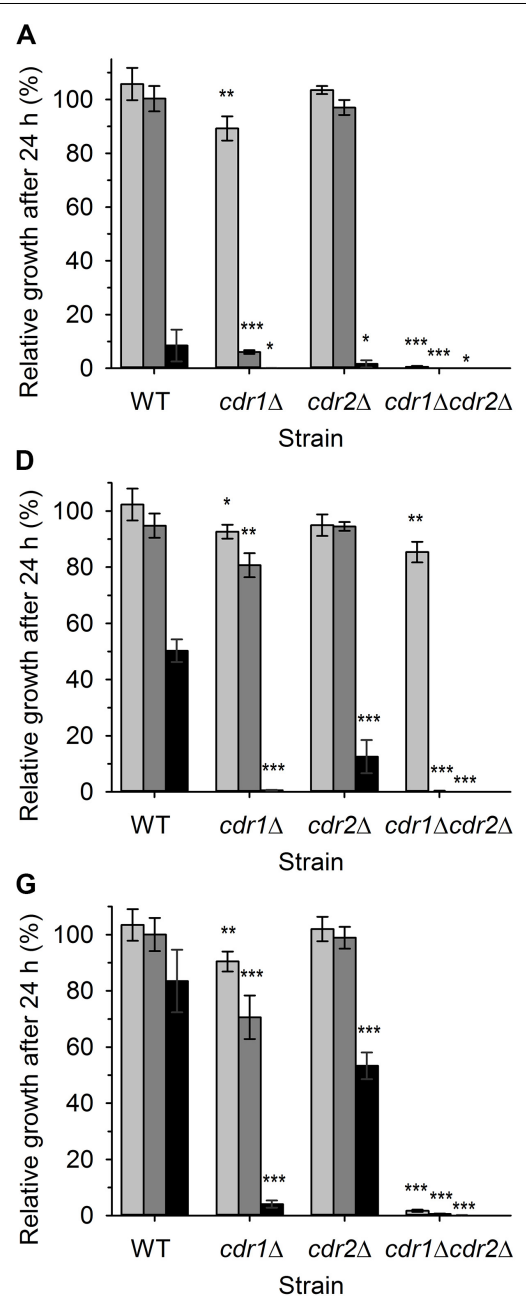

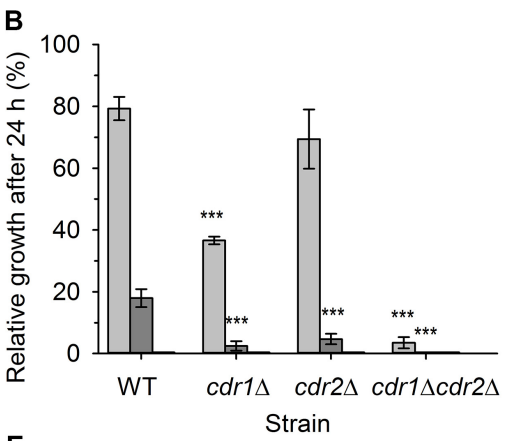

E
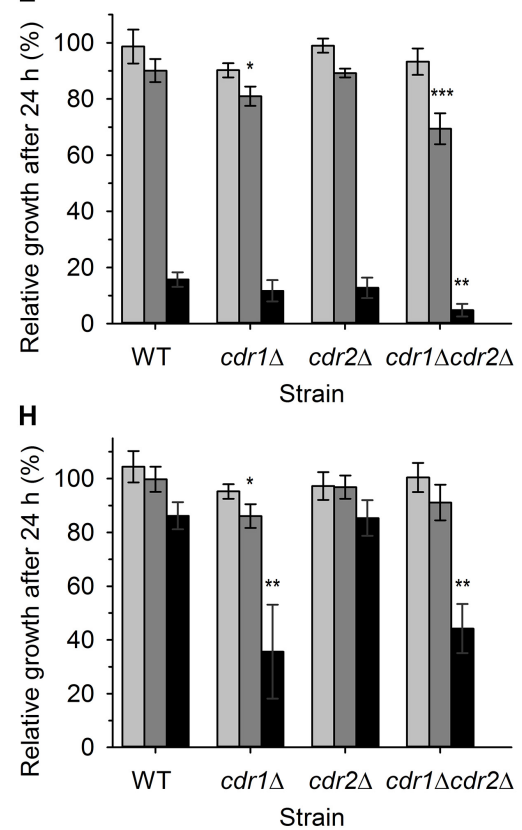
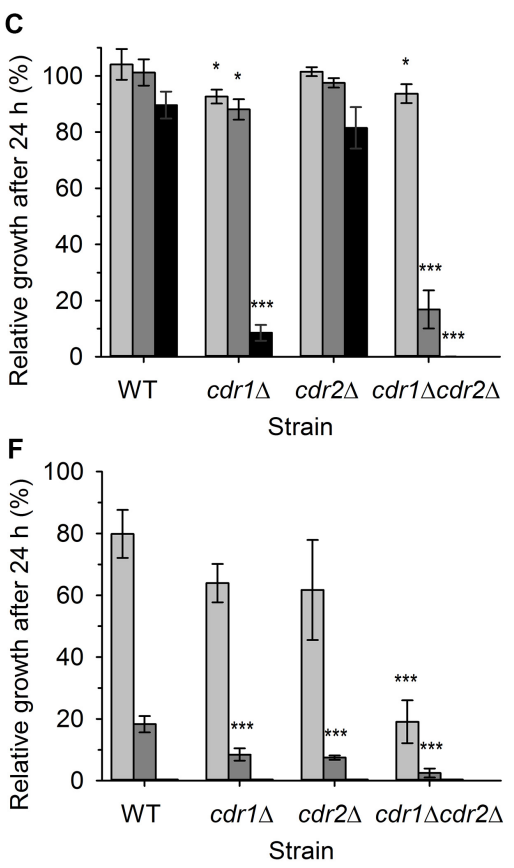

FIGURE 2 | Relative growth of four C. glabrata strains in liquid YPD medium supplemented with various concentrations of styrylpyridinium derivatives $\mathbf{1}$ (A), 2 (B), $\mathbf{3}(\mathbf{C}), \mathbf{4}$ (D), $\mathbf{5}$ (E), $\mathbf{6}$ (F), $\mathbf{7}$ (G), and $\mathbf{8}(\mathbf{H})$ in 24 h. Growth in YPD medium without any drugs was regarded as $100 \%$. (A-G) $0.5 \mu \mathrm{M}$ drugs (light gray bars), $1 \mu \mathrm{M}$ drugs (dark gray bars), $2 \mu \mathrm{M}$ drugs (black bars), (H) $5 \mu \mathrm{M}$ compound $\mathbf{8}$ (light gray bars), $10 \mu \mathrm{M}$ compound $\mathbf{8}$ (dark gray bars), $20 \mu \mathrm{M}$ compound $\mathbf{8}$ (black bars). The $P$-values $\left({ }^{\star} P<0.05,{ }^{\star \star} P<0.01,{ }^{\star \star \star} P<0.001\right)$ denote statistically significant differences from the wild-type strain.

the addition of styrylpyridinium derivatives by the diS- $\mathrm{C}_{3}(3)$ fluorescence assay.

\section{Effect of Styrylpyridinium Derivatives on the Cell Plasma-Membrane Integrity}

The diS- $\mathrm{C}_{3}(3)$ assay monitors the rate of influx of this cationic probe into cells, which is driven by the internal negative membrane potential in intact cells. A very rapid increase in staining suggests that the integrity of plasma membrane is disrupted and the probe enters cells independently of the membrane potential (Kodedová et al., 2011, 2019). Moreover, comparing the changes in staining curves of wild-type and $c d r 1 \Delta$ cells upon the addition of the tested compounds should confirm the results of the growth test, i.e., the ability of Cdr1 to eliminate the tested styrylpyridinium compounds from cells.

We tested the effects of styrylpyridines (over a broad range of concentrations from $0.5-20 \mu \mathrm{M}$ ) on the wild type and $c d r 1 \Delta$ mutant. In this screening, we had to leave out compound 1 , because it strongly interacted with the diS- $\mathrm{C}_{3}(3)$ probe and disturbed its fluorescence emission spectrum (not shown). The most interesting results obtained for compounds $\mathbf{2 - 8}$, illustrating the effect of styrylpyridinium derivatives on plasma-membrane integrity and the Cdr1-mediated efflux of these compounds, are summarized in Figure 3. Simultaneously, to distinguish between minor damage or only harmless hyperpolarization/higher staining caused by styrylpyridines and serious damage leading to the membrane permeabilization, $c d r 1 \Delta$ cell viability was estimated after 20 -min exposure to the tested compounds (Figure 4).

Figure 3A shows an example of a severe plasma-membrane damage caused by $10 \mu \mathrm{M}$ compound 2 , as well as confirming it to be a substrate of Cdr1. Control curves (empty circles) showed a higher staining of the $c d r 1 \Delta$ mutant (gray) than of the wildtype cells (black). The difference corresponds to a higher amount of the probe in $c d r 1 \Delta$ cells. Compound 2 was confirmed to 

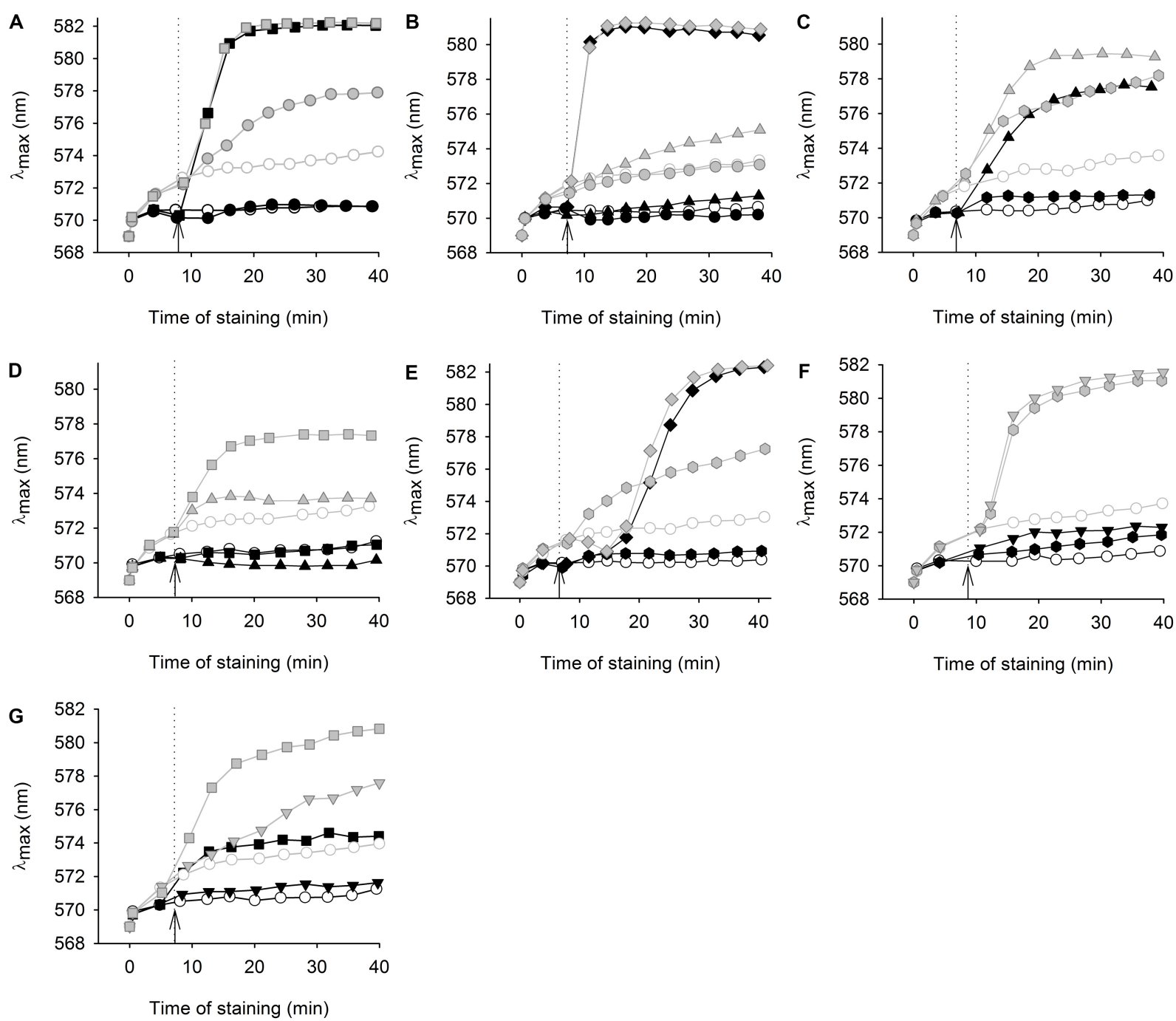

FIGURE 3 | Effect of styrylpyridinium derivatives on the staining of two C. glabrata strains with a potentiometric probe. Changes in diS- $\mathrm{C}_{3}(3)$ staining of wild-type (black symbols) and cdr1 $\Delta$ (gray symbols) cells after addition of styrylpyridinium derivatives (full symbols); (A) $1 \mu \mathrm{M}$ (circles) and $10 \mu \mathrm{M}$ (squares) compound $\mathbf{2}$, (B) $1 \mu \mathrm{M}$ (circles), $5 \mu \mathrm{M}$ (triangles) and $20 \mu \mathrm{M}$ (diamonds) compound 3, (C) $0.5 \mu \mathrm{M}$ (hexagons) and $5 \mu \mathrm{M}$ (triangles) compound 4, (D) $5 \mu \mathrm{M}$ (triangles) and $10 \mu \mathrm{M}$ (squares) compound 5, (E) $0.5 \mu \mathrm{M}$ (hexagons) and $20 \mu \mathrm{M}$ (diamonds) compound 6, (F) $0.5 \mu \mathrm{M}$ (hexagons) and $2 \mu \mathrm{M}$ (inverted triangles) compound 7, (G) $2 \mu \mathrm{M}$ (inverted triangles) and $10 \mu \mathrm{M}$ (squares) compound $\mathbf{8}$. Staining of control cells without styrylpyridine addition (empty circles); arrows with dotted lines indicate the addition of compounds.

be a substrate of Cdr1, since its presence at a relatively low concentration $(1 \mu \mathrm{M}$; full circles) led to a higher staining of $c d r 1 \Delta$ cells (Figure 3A, full gray circles), but not of wild-type cells possessing Cdr1. Brief exposure to a $1 \mu \mathrm{M}$ concentration of compound 2 did not kill $c d r 1 \Delta$ cells (Figure 4A). On the other hand, the addition of $10 \mu \mathrm{M}$ compound $\mathbf{2}$ (full squares) caused an immediate and huge increase in staining, which corresponds to a permeabilization of the membranes of both strains (Figure 3A). This rapid permeabilization resulting in the loss of viability was confirmed in the plating test (Figure 4A).

As with compound 2, we confirmed the ability of Cdr1 to also mediate the efflux of the other styrylpyridinium derivatives from C. glabrata cells (Figure 3). Differences in the effects of the individual compounds on the staining curves suggest that Cdr1 exports these compounds with different affinities. Some of the compounds, e.g., 3 and $\mathbf{4}$, significantly affected the membrane integrity at a concentration as low as $5 \mu \mathrm{M}$, and severe membrane damage was observed when the concentration of compounds was $10 \mu \mathrm{M}$ or higher (Figures 3, 4). As in the growth tests, compound $\mathbf{8}$ had the lowest negative effect of all the tested compounds. Though the presence of a $10 \mu \mathrm{M}$ concentration caused a clearly pronounced increase in the staining curve (Figure 3G), it was not accompanied by a loss of viability (Figure 4G). Also compound 7, possessing a longer alkyl chain $\mathrm{R}$ than derivative 8, was able to cause a non-lethal massive increase in staining of $c d r 1 \Delta$ cells, in fact the highest shift in staining at the lowest concentration $(0.5 \mu \mathrm{M})$ of all the tested drugs (Figure 3F). 

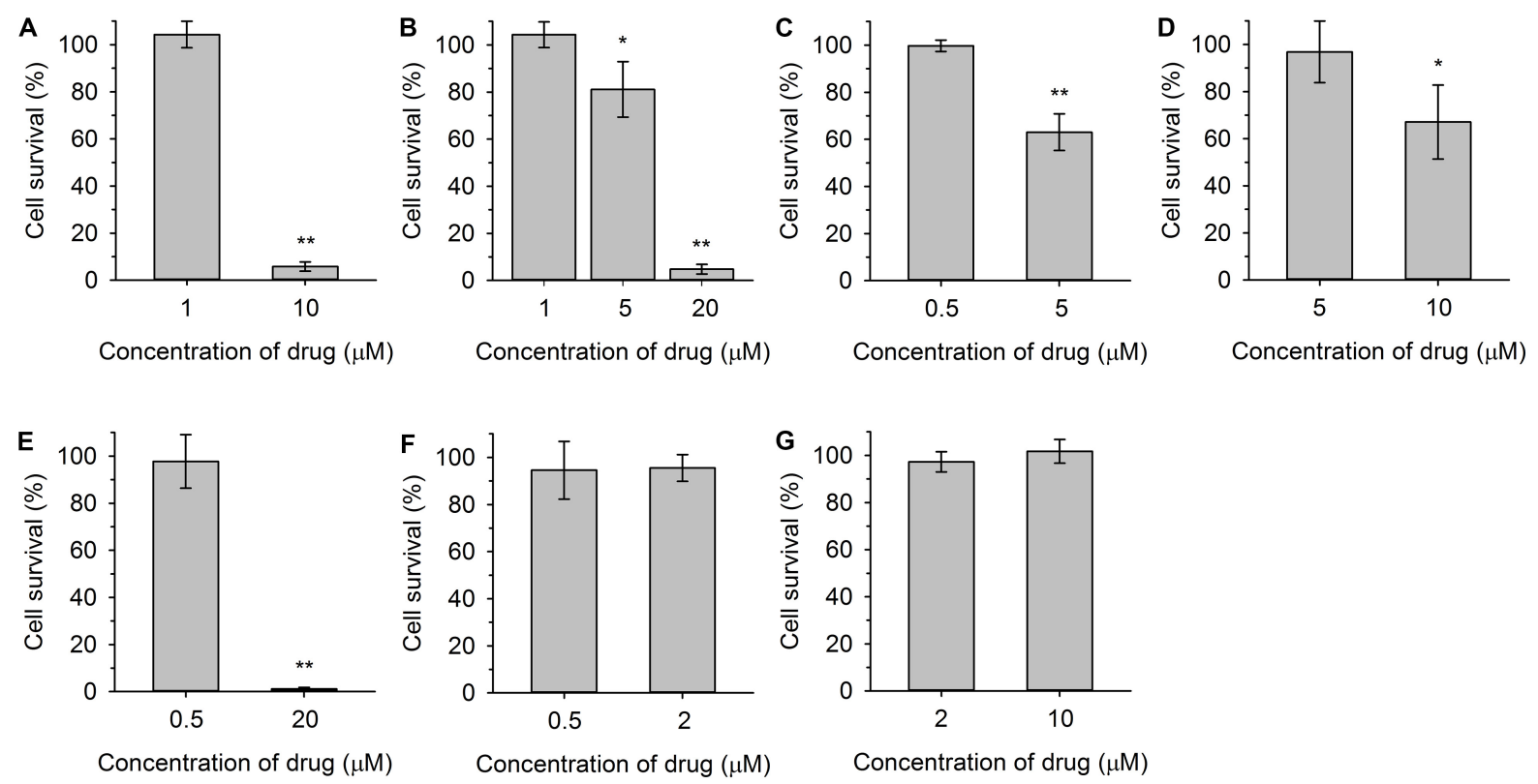

FIGURE 4 | Viability of cdr1 $\Delta$ cells after 20-min exposure to indicated concentrations of styrylpyridinium compounds 2 (A), 3 (B), 4 (C), 5 (D), 6 (E), 7 (F), and 8 (G) The control sample without exposure to the drugs was regarded as $100 \%$ cell survival. The $P$-values $\left({ }^{\star} P<0.05,{ }^{\star \star} P<0.001\right)$ denote statistically significant differences compared to the control.

Compounds 2 and $\mathbf{6}$ were the best inhibitors of growth (Figures 2B,F) and were also highly potent at causing rapid membrane damage leading to the loss of cell viability (Figures 3A,E, 4A,E). $10 \mu \mathrm{M}$ compound $\mathbf{2}$ and $20 \mu \mathrm{M}$ compound 6 completely permeabilized plasma membranes of both strains independently on the presence of Cdr1. In these cases, Cdr1 pump is no longer able to protect the cells from drugs. Nevertheless, the permeabilization of the plasma membrane with compound $\mathbf{6}$ occurred later than that caused by compound $\mathbf{2}$ (Figures 3A,E), which suggested a difference in their mode of action, and might be connected to their molecular structure. Compound 6 has a shorter hydrophobic aliphatic R substituent than compound 2 (Table 1). Compared to compounds $\mathbf{2}$ and $\mathbf{6}$, the third derivative with a hydroxyl substituent and the shortest alkyl chain (R), compound 5, produced a lower effect at the $5 \mu \mathrm{M}$ concentration (Figure 3D). In summary, this derivative was the least toxic of all the hydroxystyrylpyridines in both the short and long term (Figures 2E, 3D, 4D).

In summary, we demonstrated that the effects of styrylpyridinium derivatives on the plasma membrane integrity of C. glabrata cells were dependent on their structure and concentrations. Longer aliphatic $\mathrm{R}$ chains (Table 1) in groups of derivatives that were otherwise the same caused a stronger impairment of the plasma membrane. Analogously, permeabilizing potency decreased (higher concentration required for equivalent results) and even slowed down (longer time needed) from $2\left(-\mathrm{C}_{12} \mathrm{H}_{25}\right)$ through $6\left(-\mathrm{C}_{10} \mathrm{H}_{21}\right)$ to 5 $\left(-\mathrm{C}_{8} \mathrm{H}_{17}\right)$. The antifungal mechanism of action of the tested drugs evidently includes the incorporation of their molecules into the lipid bilayer, in which the hydrophobic alkyl chains are situated along the non-polar hydrocarbon chains of lipids and the positively charged pyridinium moiety settles in the polar environment near the plasma membrane surface. Longer tails most likely strengthen the interactions between styrylpyridines and lipids in the membrane. Since the studied compounds only contain one saturated aliphatic chain compared to the double-tailed lipids, they probably modify membrane fluidity and destabilize lipid membranes like detergents. Therefore the toxicity of the agents is dependent on cell density, because it influences the drug/membrane lipid ratio, and thus both the drug and cell concentrations determine the results. It also explains the apparent differences between toxic concentrations found in the growth tests (Figure 2) and in the estimation of cell plasma-membrane integrity (Figures 3,4), because the cell density (OD) was $200 \times$ lower at the beginning of the growth tests than in the other experiments.

Since we found that all the styrylpyridinium derivatives were transported from C. glabrata cells as substrates of its MDR pumps, they would probably not be suitable for a single-drug antifungal therapy, but they may find their applications in combined therapy together with other antifungal drugs, which are often also substrates of MDR pumps. Styrylpyridinium compounds could increase the concentration of the given drug above the effective threshold due to the competition of two drugs for the binding sites of MDR transporters.

\section{Synergistic Effect of Styrylpyridinium Compounds With Antifungal Drugs}

Combined therapy has become an important alternative for treating invasive fungal infections, leading to an improved efficacy of conventional antifungal drugs (such as azoles, 
amphotericin B or echinocandins) and at the same time reducing their side effects by using lower therapeutic doses. Several studies have shown a synergistic effect of azoles in combination with calcineurin inhibitors FK506 (tacrolimus) and cyclosporine A or other drugs (Mathé and Van Dijck, 2013; Denardi et al., 2015; Shrestha et al., 2015; Park et al., 2019). We performed disk diffusion tests to evaluate a possible synergistic effect of styrylpyridinium compounds 1-8 with FK506, terbinafine, fluconazole and ketoconazole against the four C. glabrata strains with and without Cdr pumps. Unfortunately, we did not observe any significant synergism between styrylpyridines and fluconazole or ketoconazole (data not shown), but we found strong synergism of some of the tested compounds in combination with FK506 (Figure 5) and terbinafine (Figure 6).

FK506 is a well-known and widely used immunosuppressant, which inhibits calcineurin phosphatase and prevents the T-cell activation and proliferation involved in transplant rejection. The antifungal action of FK506 is very similar to its immunosuppression mechanism, since the core components of the $\mathrm{Ca}^{2+}$-calcineurin signaling pathway, such as calmodulin and calcineurin, are conserved in eukaryotes, although the functions of these pathways differ between organisms (Cruz et al., 2002; Denardi et al., 2015; Park et al., 2019). In pathogenic fungi, calcineurin controls essential virulence pathways, such as the ability to grow at human body temperature, morphogenesis (hyphal or pseudohyphal growth), drug tolerance/resistance, and cell wall integrity (Park et al., 2019). The importance of the calcineurin pathway for fungal virulence makes its inhibitor FK506 a promising antifungal drug. Moreover, FK506 is able to act synergistically with azoles against C. albicans and C. glabrata (Cruz et al., 2002; Denardi et al., 2015).

In our case, when FK506 was applied alone at a $30 \mathrm{mM}$ concentration on paper disks, it did not form any growth inhibition zones in the four C. glabrata strains used, but we observed a pronounced effect when it was combined with compounds 5 or $\mathbf{6}$ (Figure 5). Alone, compound 5 was only able to form bland zones (slightly decreased yeast density in diffusion areas around disks, which was the most prominent with the double mutant), however its combination with FK506 gave rise to large bright zones in all C. glabrata strains (Figure 5). Compound 6 gave similar results. Although alone this drug created wider and clearer zones than compound $\mathbf{5}$, together with FK506 it made slightly smaller zones compared to compound 5. Nevertheless, compound $\mathbf{6}$ alone achieved the clearest inhibitory effect against the double mutant, and FK506 markedly strengthened the fungicidal efficiency of this drug on all the tested strains (Figure 5).

While FK506 together with styrylpyridinium compounds $\mathbf{5}$ and 6 demonstrated excellent antifungal properties, these specific combinations may have limited applicability in human medicine because of host cross-reactivity resulting from immunosuppression caused by FK506. This restriction can be overcome with non-immunosuppressive FK506 analogs which keep their antifungal potency, such as L-685,818 and 9-deoxo-31-O-demethyl-FK506 or other FK506 derivatives with modified functional groups that modulate their antifungal and immunosuppressive activities (Jung and Yoon, 2020). On the other hand, the combination of styrylpyridinium compounds and FK506 can be directly advantageous for one group of patients: organ and bone marrow transplant recipients already receive calcineurin inhibitors as part of their immunosuppressive regime to prevent graft rejection and antifungal drugs (azoles) to treat concomitant fungal infections (Cruz et al., 2002).

Further, we tested the synergism of eight styrylpyridinium compounds and terbinafine, which blocks the ergosterol biosynthetic pathway in an earlier step (Erg1) than azoles. The resulting ergosterol depletion and squalene accumulation affects membrane structure and function (Campoy and Adrio, 2017). We suppose that these changes in the lipid composition of C. glabrata membranes may enhance the killing effectivity of styrylpyridinium compounds, since some reports have already described an increased efficiency of terbinafine in combination with other antifungal drugs on C. albicans or C. dubliniensis (Barchiesi et al., 1998; Scheid et al., 2012).

In our tests, only derivatives 2, 3, 5, and $\mathbf{6}$ exhibited a significant synergistic effect in combination with terbinafine (Figure 6). Terbinafine applied alone formed quite small but bright inhibition zones for the two strains lacking Cdr1, as it is a substrate of this MDR pump (Puri et al., 2011). All of the above-mentioned hydroxy- and dimethoxystyrylpyridinium derivatives inhibited the growth of deletion mutants more than the wild type. Zones observed with $c d r 1 \Delta$ and $c d r 1 \Delta c d r 2 \Delta$ strains were more noticeable than those observed for the wildtype strain, again validating our previous conclusion that most of the tested compounds are eliminated from cells by MDR pumps, predominantly Cdr1. We observed larger growth inhibition zones formed in the presence of terbinafine combined with styrylpyridinium derivatives possessing longer aliphatic chains $\left(-\mathrm{C}_{12} \mathrm{H}_{25}\right.$ of compounds 2 and $3,-\mathrm{C}_{10} \mathrm{H}_{21}$ of drug 6) than only an octyl substituent (5). Styrylpyridinium derivatives with longer alkyl chains are probably more tightly anchored between the surrounding lipids in C. glabrata membranes, because this yeast incorporates almost identical proportions of lipids with C16 and C18 fatty acids, in contrast to other Candida species that predominantly contain C18 fatty acids in membrane lipids (Kodedová et al., 2019). Terbinafine treatment initiates a drop in C18 fatty acids content in favor of C16 and an increase in the saturation of fatty acids in C. glabrata (Kodedová et al., 2019), which could even strengthen the interaction between similarly long chains of styrylpyridinium compounds and membrane lipids.

\section{Spectral Characterization of Styrylpyridinium Compounds}

As was mentioned in the Introduction, some of the tested styrylpyridinium derivatives were originally synthesized as new fluorescence probes and their fluorescence spectra have already been published, e.g., compounds 5, 7, and 8 (Dubur et al., 1984; Krieg et al., 2007; Shiraishi et al., 2010). Some of the compounds used in this study have not yet been characterized in terms of how the length of their alkyl chain and specific substituents R1 and R2 on the aromatic ring (Figure 1 and Table 1) influence their spectroscopic properties. First, we measured the absorption 


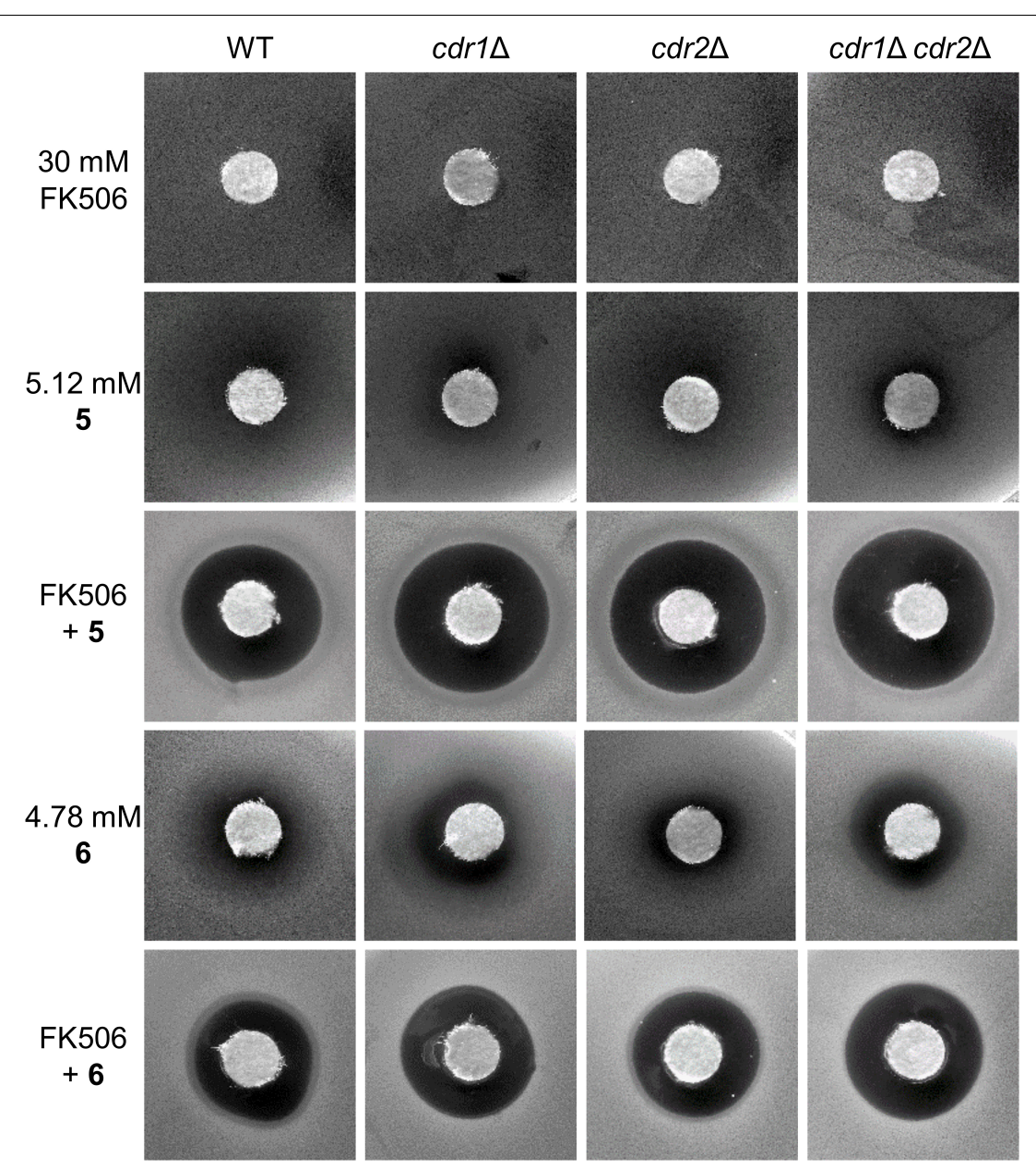

FIGURE 5 | Growth inhibition zones for C. glabrata wild type and its mutants lacking Cdr1 and/or Cdr2 pumps exposed to FK506 or styrylpyridinium derivatives $\mathbf{5}$ and $\mathbf{6}$ at indicated concentrations and their combinations.

(terbinafine
cdr1 $\Delta$
cdr2 $\Delta$



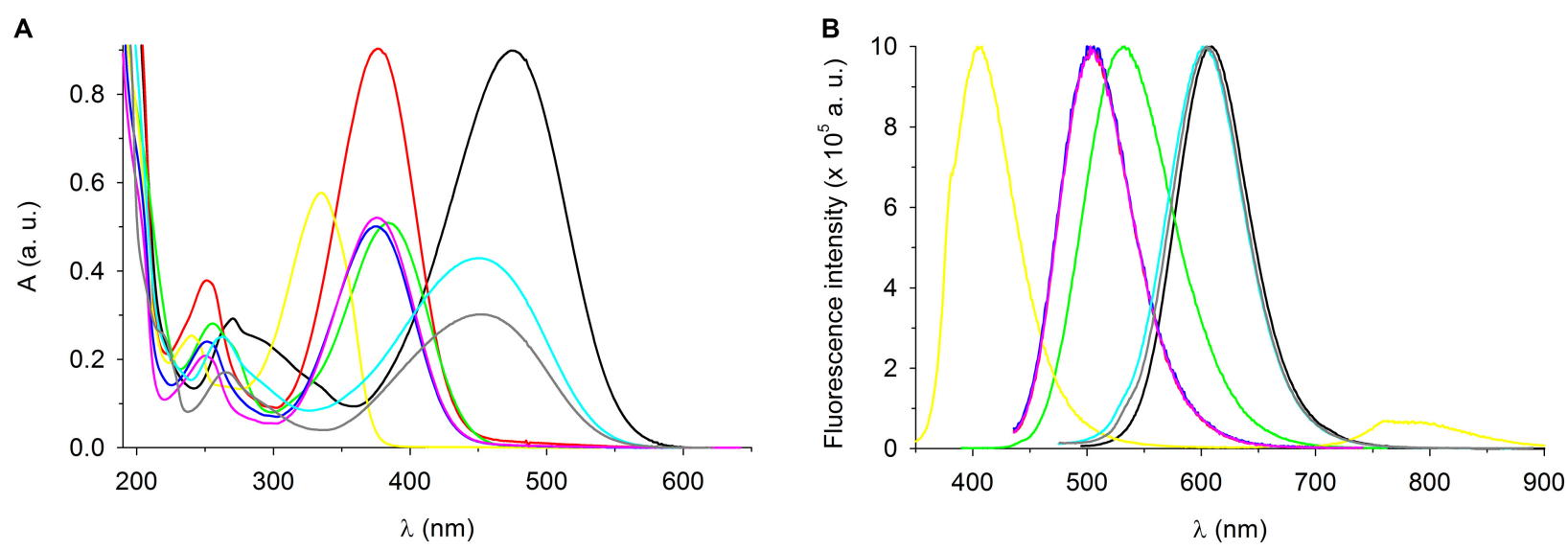

FIGURE 7 | Spectroscopic characterization of styrylpyridinium derivatives. (A) Absorption and (B) fluorescence spectra of $20 \mu \mathrm{M}$ styrylpyridinium derivatives in deionized water. Fluorescence spectra were normalized. Compound $\mathbf{1}$ (black line), $\mathbf{2}$ (red line), $\mathbf{3}$ (green line), 4 (yellow line), 5 (blue line), 6 (pink line), $\mathbf{7}$ (turquoise line), and $\mathbf{8}$ (gray line).

spectra of $20 \mu \mathrm{M}$ styrylpyridines in distilled water to find the optimal wavelengths for their excitation (Figure 7A). The absorption spectra of the whole group of drugs had the same main features, namely two major absorption peaks. The first, less intensive bands of all drugs, were found in the UV region. Their maxima were situated between $239 \mathrm{~nm}$ (compound 4) and $271 \mathrm{~nm}$ (compound 1). The second dominant absorption bands were shifted toward the visible spectrum, except for compound 4 . The entire absorption of compound $\mathbf{4}$ was clustered purely in the UV region (Figure 7A). Therefore, its solution was the only one that was colorless. Other drugs gave rise to yellow, orange or red solutions, depending on their concentrations. The maxima of these primary absorption bands (Figure 7A), summarized in Table 3, were used for the excitation of styrylpyridinium fluorescence. Normalized fluorescence spectra are shown in Figure 7B.

The shapes of all emission spectra were very similar, with full width at half maximum (FWHM) ranging from $69 \mathrm{~nm}$ (compound 4) to $91 \mathrm{~nm}$ (compound 3). The spectral halfwidth of each hydroxystyrylpyridinium derivative $(2,5$, and 6$)$ was identical $(74 \mathrm{~nm})$, indicating that this spectral parameter was evidently independent of the length of the aliphatic chain and instead defined by the R2 substituent (Table 1). This observation was also confirmed by four compounds (1-4) with the same dodecyl groups and different R2 substituents, whose emission bandwidths differed by up to $22 \mathrm{~nm}$. Not only FWHM, but also the position of the maximum (both absorption and emission) was predominantly determined by the R2 substituents on aromatic rings, as documented in Figure 7 and Table 3. All hydroxystyrylpyridines emitted maximum fluorescence at 504-505 nm, therefore their emission spectra overlapped (Figure 7B), and also the differences between the positions of the absorption maxima were minimal (Table 3). Besides hydroxystyrylpyridines $\mathbf{2 , 5}$, and $\mathbf{6}$, the second group with overlapping fluorescence spectra in Figure 7B was composed of dimethylaminostyrylpyridines $\mathbf{7}$ and $\mathbf{8}$ and accompanied by diethylaminostyrylpyridine $\mathbf{1}$. Whereas the fluorescence spectra of these three compounds were very close to each other, there were significant differences between diethylamino- (1) and dimethylaminostyrylpyridines $(7,8)$ in the shape and position of their absorption peaks (Figure 7A and Table 3). Unlike the other dyes, the emission of compound $\mathbf{4}$ was localized to the shortest wavelengths of the spectrum (maximum $\lambda_{\mathrm{em}}=405 \mathrm{~nm}$ ), and we also registered emitted light by this drug in the nearinfrared region.

The extraordinary large Stokes shifts of the tested styrylpyridines (Table 3) may be of significant benefit for their future biological applications. Common fluorophores such as fluorescein and rhodamine dyes exhibit small Stokes shifts (24-26 nm), which can reabsorb emitted photons leading to undesired background interference (Gao et al., 2017). The advantage of fluorescence dyes with large Stokes shifts (typically over $80 \mathrm{~nm}$ ) is a minimization of cross-talk between the excitation source and fluorescence emission, meaning cellular imaging with a high signal-to-noise ratio (Gao et al., 2017). Excluding compound 4, which has a Stokes shift of only $70 \mathrm{~nm}$, the remaining derivatives more than fulfill the criterion of large Stokes shifts, which lengthen from hydroxy- $(\mathbf{2}, \mathbf{6}$, and 5 with $128,129$, and $130 \mathrm{~nm})$ through diethylamino- (1 with $135 \mathrm{~nm})$ and dimethoxy- (3 with $147 \mathrm{~nm}$ ) to dimethylstyrylpiridines ( 7 and 8 with $153 \mathrm{~nm}$ ).

In summary, the small overlaps between the absorption and fluorescence spectra of all compounds (Figure 7) together with large Stokes shifts (Table 3) suggest good potential for their practical applications.

\section{Staining of C. glabrata Cells With Styrylpyridinium Derivatives}

To confirm the biological applicability of the styrylpyridinium derivatives, we used confocal fluorescence microscopy to examine the cell staining and possible preferential localization of styrylpyridinium dyes within yeast cells. We chose a 405$\mathrm{nm}$ laser line for the excitation of all compounds except for 


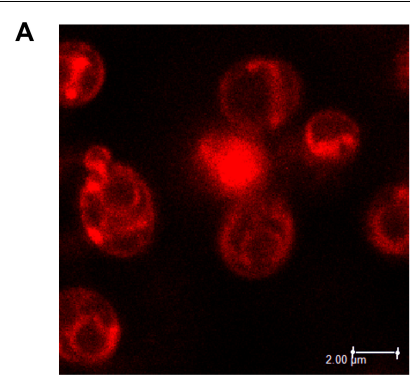

C

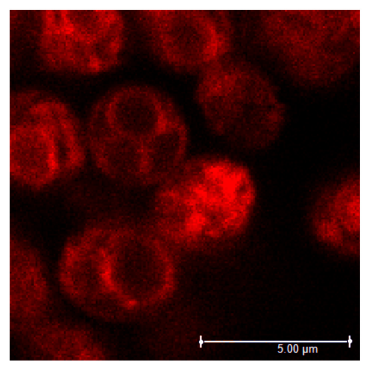

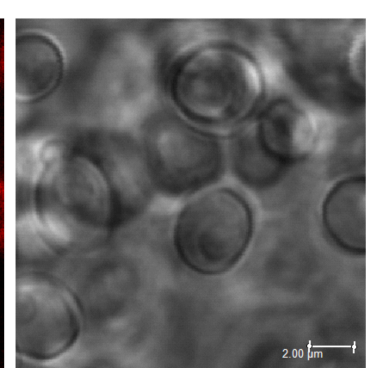

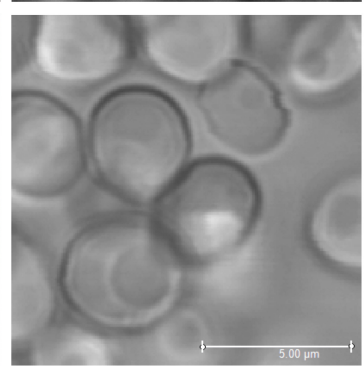

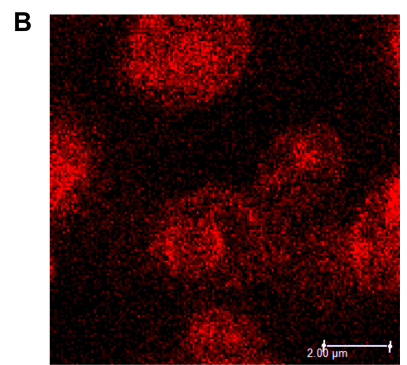

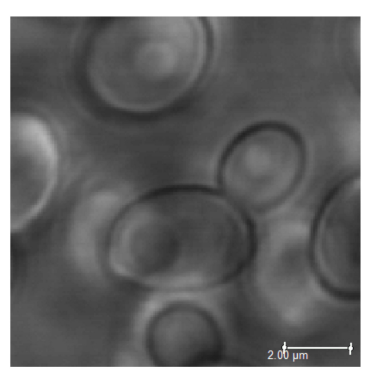

D

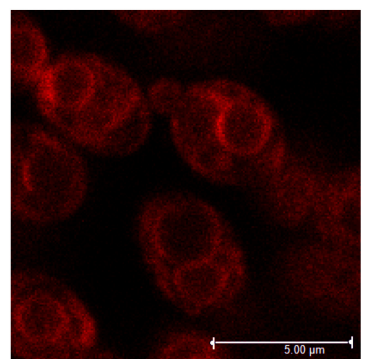

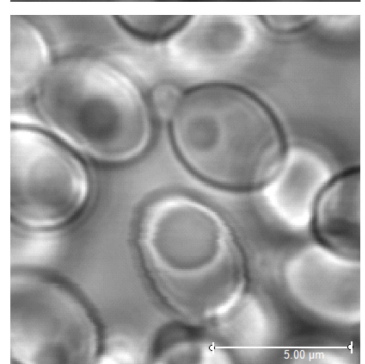

FIGURE $\mathbf{8}$ | Staining of vacuolar membranes of C. glabrata wild-type cells with styrylpyridinium derivatives $\mathbf{1}$ (A), $\mathbf{3}$ (B), $\mathbf{7}$ (C), and $\mathbf{8}$ (D). Scale bar: $2 \mu \mathrm{m}$ (A,B) or 5 $\mu \mathrm{m}$ (C,D).

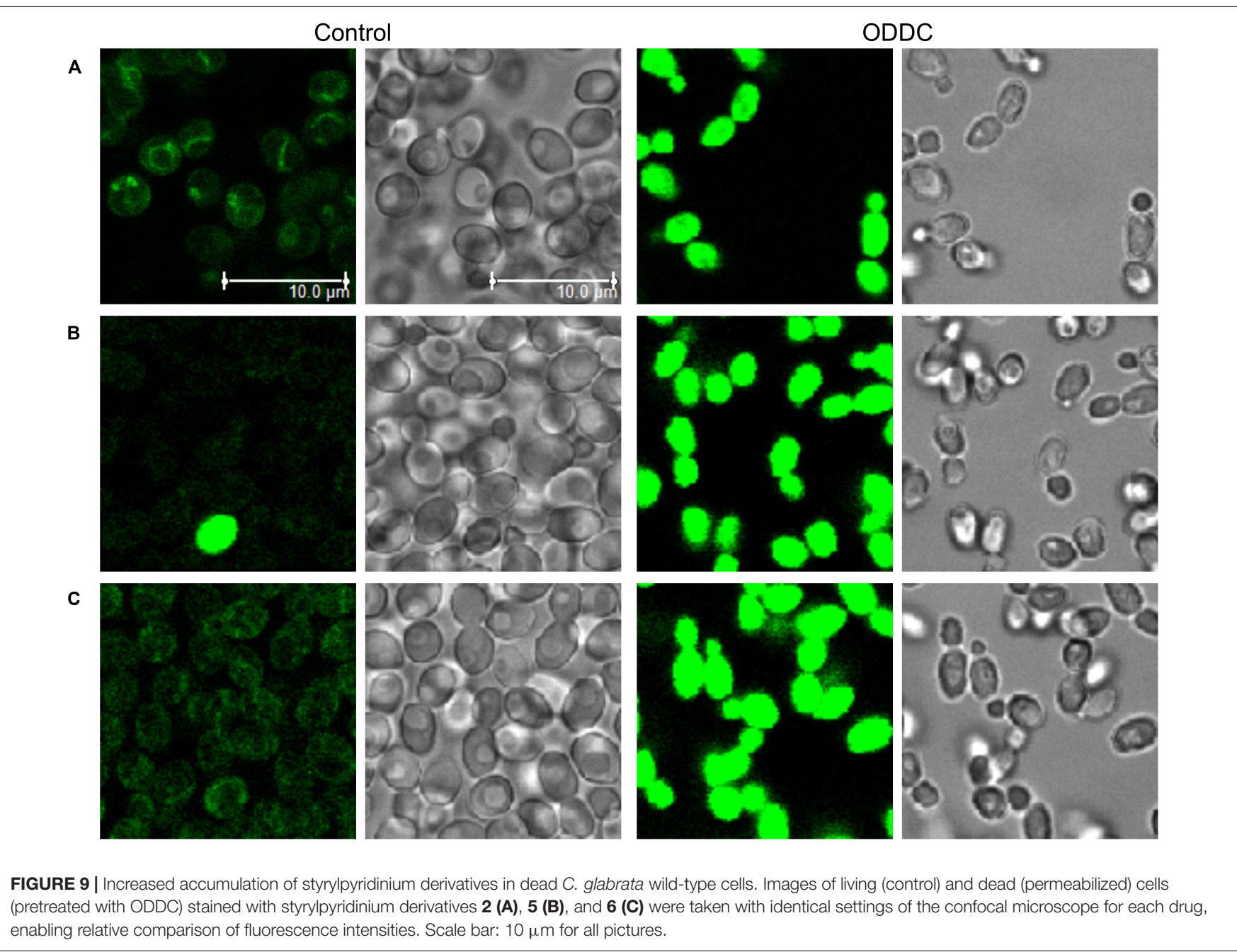


derivative 4, which did not absorb light in this spectral region (its excitation was unfeasible under our conditions) and a 2 $\mu \mathrm{M}$ concentration, which did not seriously affect the membrane integrity of wild-type cells during short exposure (Figure 3) and corresponds to the concentration usually used for the commercial styrylpyridinium fluorescence dye FM4-64 (Miner et al., 2019). According to the distribution of fluorescence in C. glabrata wildtype cells, we can divide the tested compounds into two groups. The first group, comprised of compounds 1 (Figure 8A), 3 (Figure 8B), 7 (Figure 8C), and 8 (Figure 8D), was able to stain yeast plasma and vacuolar membranes, which exhibited higher fluorescence intensity compared to the cytoplasm with only dispersed fluorescence. With these compounds, we could observe several distinct vacuoles in each yeast cell (Figure 8), which is typical for an exponentially growing yeast culture. All derivatives with this specific distribution contained diethylamino-, dimethylamino- or dimethoxy- substituents, which evidently played analogous roles in the interaction with membrane structures such as the dibutylamino- group of the commercial styrylpyridinium vacuolar marker FM4-64. It is widely believed that FM4-64 and FM1-43 enter the cell primarily via endocytic vesicles invaginated from the plasma membrane and they insert into the membrane lipid bilayer via their lipophilic tails with the pyridinium dicationic head anchored at the membrane surface (Bolte et al., 2004). We suppose that the tested styrylpyridinium derivatives $\mathbf{1}, \mathbf{3}, \mathbf{7}$, and $\mathbf{8}$ share enough essential molecular features with commercial styrylpyridinium dyes and utilize a similar entrance mechanism via endocytosis. However further studies will be necessary to prove this assumption.

The second group of derivatives (2, 5, and 6) were not accumulated in any particular compartment of yeast cells (Figure 9). They stained whole yeast cells more homogenously. However, we discovered a striking difference between the fluorescence intensities of living and dead cells, e.g., one damaged cell visualized by compound $\mathbf{5}$ among living cells (Figure 9B). To confirm that these three compounds could be used as vital dyes, we treated C. glabrata wild-type cells with $3 \mu \mathrm{M}$ octenidine dihydrochloride (ODDC) for $15 \mathrm{~min}$, causing full permeabilization of their plasma membranes (Kodedová et al., 2019). These pretreated and washed cells were used as an example of killed cells for styrylpyridinium staining. We observed (Figure 9) a massive increase in the fluorescence intensity of dead cells stained by all three derivatives compared to the living (control) cells, which exhibited very low emission under identical settings of the confocal microscope for each drug, enabling a relative comparison of fluorescence intensities. Thus, depending on the structure of molecules, styrylpyridines can find their application as vital dyes (Figure 9) or vacuolar membrane probes (Figure 8).

We excited all styrylpyridinium compounds with a 405-nm laser, which is frequently used for the excitation of nuclearspecific DAPI or Hoechst dyes. But in contrast to these DNA binding probes, the emission of styrylpyridines is shifted toward longer wavelengths as indicated by their unusually large Stokes shifts. This fact makes them suitable candidates for the multicolor staining of cells. Moreover, compounds 1, 7, and 8 can be also very effectively excited at 440 or $473 \mathrm{~nm}$. These $\lambda_{\text {ex }}$ are often utilized for the excitation of the fluorescence proteins CFP and GFP. Compared to these cyan and green fluorescence proteins, the fluorescence of styrylpyridinium derivatives 1, 7, and 8 can be clearly detected in the red channel (>650 nm) and thus enables co-localization studies with CFP- or GFP-fused cell proteins. Simultaneously, our styrylpyridinium derivatives provide spectral alternatives to current tonoplast markers, because FM-dyes are excited by longer-wavelength lasers such as 488,514 , or $532 \mathrm{~nm}$ (Bolte et al., 2004).

Likewise, fluorescence microscopy clearly showed cell membranes to be the main target of styrylpyridinium compounds. Their antifungal activity, consisting of the permeabilization of the plasma membrane, was strongly dependent not only on exposure time, but also on achieving the threshold concentrations when they start to behave as detergents. Therefore the actual ratio of styrylpyridinium molecules per yeast cell seems to be crucial for the final effect, and a $2 \mu \mathrm{M}$ concentration could be harmless during short-term exposures of abundant cells such as microscopy staining or the estimation of cell plasma-membrane integrity in contrast to the long-term exposure of the several hundred times smaller cell population in a growth test.

\section{Styrylpyridinium Derivatives as New Fluorescence Benchmark Substrates of C. glabrata MDR Pumps}

We were interested in possible application of styrylpyridinium compounds as new fluorescence substrates of C. glabrata Cdr1 or Cdr2 pumps for monitoring efflux via these transporters. These drugs could not only enlarge a small group of fluorescence substrates suitable for study of C. glabrata MDR pumps, but also due to their completely different molecular structures from commonly used rhodamine $6 \mathrm{G}$ or diS- $\mathrm{C}_{3}(3)$ probes, styrylpyridines can offer new information about substrate

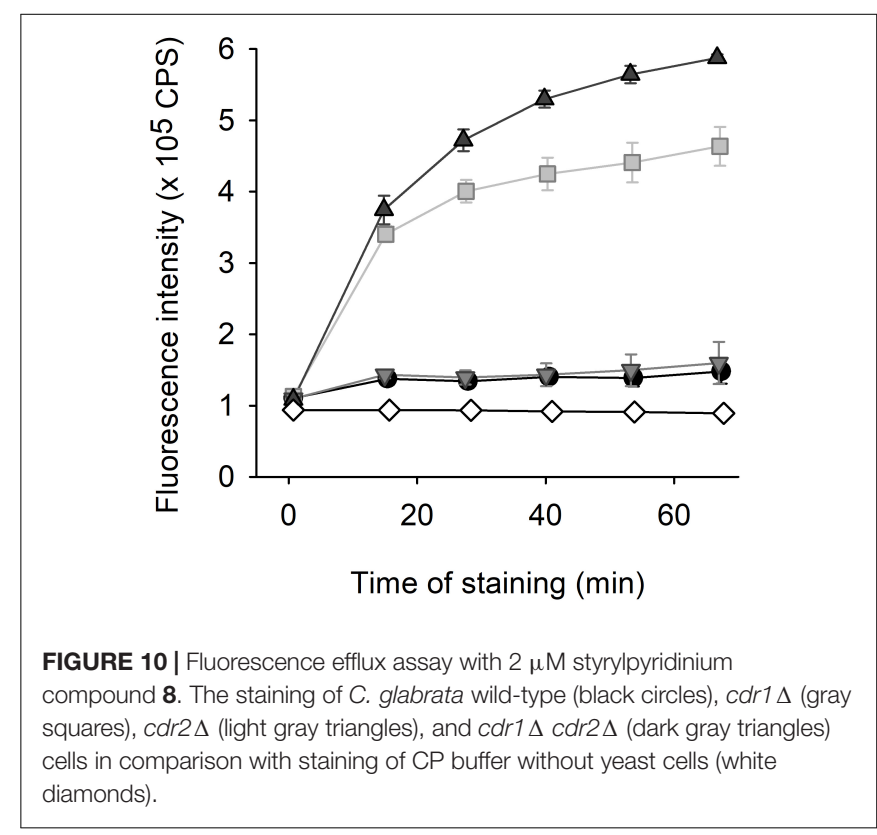


transport by $C g \mathrm{Cdr} 1$ and $C g \mathrm{Cdr} 2$. For this purpose, we select compound $\mathbf{8}$ as the least toxic derivative.

Compound 8 responded to changes in its environment by altering its spectral characteristics. Maximum of its fluorescence emission measured in yeast cell suspension was shifted (in the range of several nanometers) to shorter wavelengths in comparison with the position in $\mathrm{CP}$ buffer or distilled water (data not shown), simultaneously, the intensity of fluorescence emission increased more considerably. Thus, we chose this spectral parameter for design of fluorescence efflux assay of compound $\mathbf{8}$ (Figure 10). The compound $\mathbf{8}$ exhibited a timeand strain-dependent accumulation in yeast cells accompanied by an increase in emission intensity. Strains lacking Cdr1 pump accumulated several times higher amount of the dye compared to the wild type. The difference between staining curves of $c d r 1 \Delta$ and $c d r 1 \Delta c d r 2 \Delta$ strains suggested that also Cdr2 pump was able to eliminate compound $\mathbf{8}$ from $C$. glabrata cells. The negligible difference between wild-type and $c d r 2 \Delta$ cells was probably given by dominant role of CDR1 due to its overexpression in these strains (unlike basal level of CDR2).

It was the first layout of the transport assay with styrylpyridinium compound (Figure 10) and several parameters would need to be optimized for routine applications. Nevertheless, according to our opinion, compound $\mathbf{8}$ has the greatest potential to become a new benchmark fluorescence substrate for finding new inhibitors of $C g C d r 1$ as the least toxic styrylpyridinium derivative. Although we obtained similar results with its close derivative, compound 7 (data not shown), its higher antifungal activity could be undesirable in this application (analogously other derivatives that possess high antifungal activity).

\section{CONCLUSION}

In summary, styrylpyridinium compounds may find their applications as new antifungal drugs or fluorescence dyes in yeast research. Compounds 2 (bromide of (E)-N-dodecyl$\gamma$-azastilbenol-4') and $\mathbf{6}$ (N-decyl 4-( $p$-hydroxy styryl) pyridinium bromide) exhibited the strongest inhibition of C. glabrata growth, followed by derivatives $\mathbf{1 , 5}, \mathbf{4}, \mathbf{3}$, and 7 . The most promising antifungal agent is probably derivative $\mathbf{6}$, which was able to effectively permeabilize the plasma membrane of yeast cells on its own and also exhibited significant synergism together with FK506 or terbinafine. On the other hand, the low toxicity of some derivatives could be beneficial for their application as fluorescence dyes, minimizing their side effects on yeast morphology. The least toxic derivative 8 (N-hexyl

\section{REFERENCES}

Barchiesi, F., Di Francesco, L. F., Compagnucci, P., Arzeni, D., Giacometti, A., and Scalise, G. (1998). In-vitro interaction of terbinafine with amphotericin B, fluconazole and itraconazole against clinical isolates of Candida albicans. J. Antimicrob. Chemother. 41, 59-65. doi: 10.1093/jac/4 1.1.59 4'-dimethylaminostyryl pyridinium tosylate) could serve as a new vacuolar marker, as its spectroscopic properties differ from current tonoplast markers and enable multi-color staining due to its exceptionally large Stokes shift $(153 \mathrm{~nm})$. Moreover, this compound can diversify the range of fluorescence benchmark substrates of C. glabrata Cdr pumps. The styrylpyridinium derivative 5 (N-octyl $p$-hydroxystyrylpyridinium bromide) can be used as new fluorescence vital dye, as it accumulates rapidly in dead cells and has a low killing potential.

\section{DATA AVAILABILITY STATEMENT}

The raw data supporting the conclusions of this article will be made available by the authors, without undue reservation.

\section{AUTHOR CONTRIBUTIONS}

SV and MK performed the experiments and analyzed the data. MK contributed to the conception of the study and designed the experiments. RD and HS supervised the study. SV, MK, and HS wrote the manuscript. All authors read and approved the final manuscript.

\section{FUNDING}

This work was supported by the Ministry of Education, Youth and Sports of the CR within the framework of LQ1604 National Sustainability Program II (Project BIOCEV-FAR) and by the project "BIOCEV" (CZ.1.05/1.1.00/02.0109). The research was also funded by the European Social Fund under "The Development of Competences of Scientists, other Researchers and Students through Practical Research Activities" measure (No. 09.3.-LMT-K-712-02-0200) under a grant agreement with the Research Council of Lithuania (LMTLT).

\section{ACKNOWLEDGMENTS}

We wish to thank Prof. Gunars Duburs for kindly providing styrylpyridinium compounds and Prof. Dominique Sanglard for C. glabrata strains. We acknowledge the Imaging Methods Core Facility at BIOCEV, institution supported by the Czech-BioImaging large RI projects (LM2015062 and CZ.02.1.01./0.0/0.0/16_013/0001775, funded by MEYS CR) for their support with obtaining the imaging data presented in this manuscript. B. (2004). FM-dyes as experimental probes for dissecting vesicle trafficking in living plant cells. J. Microsc. 214, 159-172.

Campoy, S., and Adrio, J. L. (2017). Antifungals. Biochem. Pharmacol. 133, 86-96. Cannon, R. D., Lamping, E., Holmes, A. R., Niimi, K., Baret, P. V., Keniya, M. V., et al. (2009). Efflux-mediated antifungal drug resistance. Clin. Microbiol. Rev. $22,291-321$. 
Chanawanno, K., Chantrapromma, S., Anantapong, T., Kanjana-Opas, A., and Fun, H. K. (2010). Synthesis, structure and in vitro antibacterial activities of new hybrid disinfectants quaternary ammonium compounds: Pyridinium and quinolinium stilbene benzenesulfonates. Eur. J. Med. Chem. 45, 4199-4208. doi: 10.1016/j.ejmech.2010.06.014

Cruz, M. C., Goldstein, A. L., Blankenship, J. R., Del Poeta, M., Davis, D., Cardenas, M. E., et al. (2002). Calcineurin is essential for survival during membrane stress in Candida albicans. EMBO J. 21, 546-559. doi: 10.1093/emboj/21.4.546

Denardi, L. B., Mario, D. A. N., Loreto, E. S., Santurio, J. M., and Alves, S. H. (2015). Synergistic effects of tacrolimus and azole antifungal compounds in fluconazole-susceptible and fluconazole-resistant Candida glabrata isolates. Braz. J. Microbiol. 46, 125-129. doi: 10.1590/s1517-8382461201 20442

Denksteinová, B., Gášková, D., Heřman, P., Večeř, J., Malínský, J., Plášek, J., et al. (1997). Monitoring of membrane potential changes in S. cerevisiae by diS-C $\mathrm{C}_{3}$ (3) fluorescence. Folia Microbiol. 42, 221-224. doi: 10.1007/bf0281 8983

Dubur, G. Y., Dobretsov, G. E., Deme, A. K., Dubure, R. R., Lapshin, E. N., and Spirin, M. M. (1984). Fluorescent probes based on styrylpyridinium derivatives: optical properties and membrane binding. J. Biochem. Biophys. Methods 10, 123-134. doi: 10.1016/0165-022x(84)90032-0

Gao, Z., Hao, Y., Zheng, M., and Chen, Y. (2017). A fluorescent dye with large Stokes shift and high stability: synthesis and application to live cell imaging. RSC Adv. 7, 7604-7609. doi: 10.1039/c6ra27547h

Gášková, D., Brodská, B., Heřman, P., Večeř, J., Malínský, J., Sigler, K., et al. (1998). Fluorescent probing of membrane potential in walled cells: diS-C $\mathrm{C}_{3}(3)$ assay in Saccharomyces cerevisiae. Yeast 14, 1189-1197. doi: 10.1002/(sici)10970061(19980930)14:13<1189::aid-yea320>3.0.co;2-k

Heese-Peck, A., Pichler, H., Zanolari, B., Watanabe, R., Daum, G., and Riezman, H. (2002). Multiple functions of sterols in yeast endocytosis. Mol. Biol. Cell 13, 2664-2680. doi: 10.1091/mbc.e02-04-0186

Jung, J. A., and Yoon, Y. J. (2020). Development of non-immunosuppressive FK506 derivatives as antifungal and neurotrophic agents. J. Microbiol. Biotechnol. 30, 1-10. doi: 10.4014/jmb.1911.11008

Kodedová, M., Sigler, K., Lemire, B. D., and Gášková, D. (2011). Fluorescence method for determining the mechanism and speed of action of surfaceactive drugs on yeast cells. BioTechniques 50, 58-63. doi: 10.2144/00011 3568

Kodedová, M., and Sychrová, H. (2016). High-throughput fluorescence screening assay for the identification and comparison of antimicrobial peptides' activity on various yeast species. J. Biotechnol. 233, 26-33. doi: 10.1016/j.jbiotec.2016. 06.023

Kodedová, M., Valachovič, M., Csáky, Z., and Sychrová, H. (2019). Variations in yeast plasma-membrane lipid composition affect killing activity of three families of insect antifungal peptides. Cell. Microbiol. 21, e13093. doi: 10.1111/ cmi. 13093

Krieg, R., Eitner, A., Günther, W., and Halbhuber, K. J. (2007). Optimization of heterocyclic 4-hydroxystyryl derivatives for histological localization of endogenous and immunobound peroxidase activity. Biotech. Histochem. 82, 235-262. doi: 10.1080/10520290701714013

Ksiezopolska, E., and Gabaldón, T. (2018). Evolutionary emergence of drug resistance in Candida opportunistic pathogens. Genes 9:461. doi: 10.3390/ genes 9090461

Mathé, L., and Van Dijck, P. (2013). Recent insights into Candida albicans biofilm resistance mechanisms. Curr. Genet. 59, 251-264. doi: 10.1007/s00294-0130400-3

Miner, G. E., Sullivan, K. D., Zhang, C., Hurst, L. R., Starr, M. L., Rivera-Kohr, D. A., et al. (2019). Copper blocks V-ATPase activity and SNARE complex formation to inhibit yeast vacuole fusion. Traffic 20, 841-850.
Mishra, B. K., Behera, P. K., and Rath, R. (2001). Acid dissociation constants of $\mathrm{N}$-alkyl p/o-hydroxy styryl pyridinium dyes in surfactant solutions. Indian J. Chem. 40A, 155-158.

Pais, P., Galocha, M., Viana, R., Cavalheiro, M., Pereira, D., and Teixeira, M. C. (2019). Microevolution of the pathogenic yeasts Candida albicans and Candida glabrata during antifungal therapy and host infection. Microb. Cell 6, 142-159. doi: $10.15698 / \mathrm{mic} 2019.03 .670$

Park, H. S., Lee, S. C., Cardenas, M. E., and Heitman, J. (2019). Calciumcalmodulin-calcineurin signaling: a globally conserved virunce cascade in eukaryotic microbial pathogens. Cell Host Microbe 26, 453-462. doi: 10.1016/j. chom.2019.08.004

Petrikkos, G., and Skiada, A. (2007). Recent advances in antifungal chemotherapy. Int. J. Antimicrob. Agents 30, 108-117. doi: 10.1016/j.ijantimicag.2007.03.009

Puri, N., Manoharlal, R., Sharma, M., Sanglard, D., and Prasad, R. (2011). Overcoming the heterologous bias: an in vivo functional analysis of multidrug efflux transporter, CgCdrlp in matched pair clinical isolates of Candida glabrata. Biochem. Biophys. Res. Commun. 404, 357-363. doi: 10.1016/j.bbrc. 2010.11.123

Sanglard, D., Ischer, F., and Bille, J. (2001). Role of ATP-binding-cassette transporter genes in high-frequency acquisition of resistance to azole antifungals in Candida glabrata. Antimicrob. Agents Chemother. 45, 1174-1183. doi: 10.1128/aac.45.4.1174-1183.2001

Sanglard, D., Ischer, F., Calabrese, D., Majcherczyk, P. A., and Bille, J. (1999). The ATP binding cassette transporter gene CgCDR1 from Candida glabrata is involved in the resistance of clinical isolates to azole antifungal agents. Antimicrob. Agents Chemother. 43, 2753-2765. doi: 10.1128/aac.43.11.2753

Scheid, L. A., Mario, D. A. N., Kubiça, T. F., Santurio, J. M., and Alves, S. H. (2012). In vitro activities of antifungal agents alone and in combination against fluconazole-susceptible and -resistant strains of Candida dubliniensis. Braz. J. Infect. Dis. 16, 78-81. doi: 10.1590/s1413-86702012000100014

Shiraishi, Y., Inoue, T., and Hirai, T. (2010). Local viscosity analysis of triblock copolymer micelle with cyanine dyes as a fluorescent probe. Langmuir 26, 17505-17512. doi: 10.1021/la1028993

Shrestha, S. K., Fosso, M. Y., and Garneau-Tsodikova, S. (2015). A combination approach to treating fungal infections. Sci. Rep. 5:17070. doi: 10.1038/ srep 17070

Urai, M., Kaneko, Y., Niki, M., Inoue, M., Tanabe, K., Umeyama, T., et al. (2014). Potent drugs that attenuate anti-Candida albicans activity of fluconazole and their possible mechanisms of action. J. Infect. Chemother. 20, 612-615. doi: 10.1016/j.jiac.2014.06.004

Vaitkienė, S., Kuliešinė, N., Sakalauskaitė, S., Bekere, L., Krasnova, L., Vigante, B., et al. (2020). Antifungal activity of styrylpyridinium compounds against Candida alicans. Chem. Biol. Drug Des. doi: 10.1111/cbdd.13777 Online ahead of print

Wyrzykiewicz, E., Prukała, W., and Kędzia, B. (1994). Synthesis and antimicrobial properties of $\mathrm{N}$-substituted derivatives of (E)- $\alpha$-(or $\gamma)$-azastilbenols. Farmaco $49,127-131$.

Conflict of Interest: The authors declare that the research was conducted in the absence of any commercial or financial relationships that could be construed as a potential conflict of interest.

Copyright (c) 2020 Vaitkienè, Daugelavičius, Sychrová and Kodedová. This is an open-access article distributed under the terms of the Creative Commons Attribution License (CC BY). The use, distribution or reproduction in other forums is permitted, provided the original author(s) and the copyright owner(s) are credited and that the original publication in this journal is cited, in accordance with accepted academic practice. No use, distribution or reproduction is permitted which does not comply with these terms. 